\title{
Comparative Evaluation of Efficacy of "Green Tea" and "Green Tea with Xylitol" Mouthwashes on the Salivary Streptococcus mutans and Lactobacillus Colony Count in Children: A Randomized Clinical Trial
}

\author{
Maryam Hajiahmadi ${ }^{1}$, Afsaneh Yegdaneh ${ }^{2}$, Alireza Homayoni ${ }^{3}$, Hossein Parishani ${ }^{4}$, Hadi Moshkelgosha ${ }^{5}$, \\ Reza Salari-Moghaddam ${ }^{6}$
}

\begin{abstract}
Aim: Green tea is an antimicrobial agent that has beneficial effects on oral and dental health. The aim of this study was to compare the effect of "green tea" and "green tea with xylitol" mouthwashes on the salivary Streptococcus mutans and Lactobacillus colony count in children.

Materials and methods: In this double-blind randomized controlled clinical trial, 64 children aged 6-12 years were randomly divided into two groups. Subjects were instructed to wash their mouth with 5\% "green tea" or 20\% "green tea with xylitol" mouthwashes twice a day for a period of 2 weeks. Salivary counts of bacteria were determined at the baseline and after 2 weeks of intervention.

Results: A significant difference was found between the average number of bacterial colonies between the two groups after intervention $(p<0.001)$.

Conclusion: Findings of this study suggest that the effect of the "green tea with xylitol" mouthwash on reducing the number of salivary colonies of S. mutans and Lactobacillus is significantly higher than that of the "green tea" mouthwash.

Clinical significance: The green tea mouthwash can be advised for dental and oral health of children.

Keywords: Green tea, Lactobacillus, Mouthwash, Streptococcus mutans, Xylitol.

The Journal of Contemporary Dental Practice (2019): 10.5005/jp-journals-10024-2652
\end{abstract}

\section{INTRODUCTION}

Dental caries is a common chronic disease caused by the interaction between several factors including oral flora, anatomical characteristics of teeth, and dietary intakes. Streptococcus mutans is among the major factors responsible for dental caries. Lactobacillus and actinomycosis also contribute to the development of caries. ${ }^{1-3}$ Several studies have shown that salivary contamination with these microorganisms is directly associated with the risk of caries in children, adolescents, and adults, and suppression of S. mutans significantly decreases the risk of developing this condition. ${ }^{4,5}$

The pathogenesis of dental caries comprises of three stages: (1) bacterial binding to the tooth surface, (2) effect of the glycosyltransferase enzyme on sucrose and formation of glycocalyx, and (3) biofilm (plaque) accumulation, ${ }^{6}$ that acid production continues by Streptococcus and Lactobacillus in these plaques.

Given that plaque-related diseases tend to remain localized rather than invasive, the topical use of antimicrobial agents is more effective than prescribing systemic agents. ${ }^{7}$ The application of antimicrobial agents for dental caries treatment has been studied for more than five decades. ${ }^{8,9}$ Xylitol, an alcoholic sweetener not fermented by oral bacteria, is one of the antimicrobial agents. Xylitol neutralizes oral $\mathrm{PH}$ reduction and exerts a positive effect on oral health. Regular consumption of adequate dose of xylitol reduces S. mutans in plaque and saliva. ${ }^{10-13}$ Xylitol is metabolized into the cell by $S$. mutans through the fructose phosphotransferase system. Then, xylitol is metabolized to xylitol-5-phosphate, which is not only unable to use the bacteria but also can be toxic for the bacteria. ${ }^{12}$ The sweetener sensation, resulting from the use of xylitol, stimulates mouth to release saliva, which acts as a buffering
${ }^{1,6}$ Department of Pedodontics, School of Dentistry, Isfahan University of Medical Sciences, Isfahan, Iran

${ }^{2}$ Department of Pharmacognosy, School of Pharmacy, Isfahan University of Medical Sciences, Isfahan, Iran

${ }^{3}$ Department of Pharmaceutics, School of Pharmacy and Novel Drug Delivery Systems Research Center, Isfahan University of Medical Sciences, Isfahan, Iran

${ }^{4}$ Students Research Committee, School of Dentistry, Isfahan University of Medical Sciences, Isfahan, Iran

${ }^{5}$ Department of Oral and Maxillofacial Surgery, School of Dentistry, Isfahan University of Medical Sciences, Isfahan, Iran

Corresponding Author: Reza Salari-Moghaddam, Department of Pedodontics, School of Dentistry, Isfahan University of Medical Sciences, Isfahan, Iran, Phone: +98 9133257919, e-mail: reza.salari70@ yahoo.com

How to cite this article: Hajiahmadi $M$, Yegdaneh $A$, Homayoni $A$, et al. Comparative Evaluation of Efficacy of "Green Tea" and "Green Tea with Xylitol" Mouthwashes on the Salivary Streptococcus Mutans and Lactobacillus Colony Count in Children: A Randomized Clinical Trial. J Contemp Dent Pract 2019;20(10):1190-1194.

Source of support: Isfahan University of Medical Sciences, Isfahan, Iran

Conflict of interest: None

system against the acidic environment produced by dental plaque microorganisms, resulting in neutralized plaque $\mathrm{PH} .{ }^{14}$ Therefore, xylitol induces demineralized enamel deeper layers by facilitating calcium movement and increasing its access. ${ }^{15}$ 
On the other hand, green tea has received growing attention due to its clinical features including its impact on the risk of cardiovascular diseases, stroke, obesity, and cancer. ${ }^{16-19}$ Studies have shown that bioactive components of green tea can affect the formation of caries in different ways. Green tea can prevent tooth decay by preventing the proliferation of streptococcal agents, interfering with the enamel bonding process or inhibiting the glycosyltransferase bacteria enzyme. The main component of green tea is polyphenols, especially flavonoids such as proanthocyanidin, catechin, catechin proanthocyanidin, and cathechin gallate. Most of the beneficial effects of green tea are related to its catechin content. Studies have shown that catechins prevent the attachment of oral streptococcus to the tooth surface, due to the denaturation of fibrils and bacterial fimbriae. ${ }^{20}$ Previous studies have documented the beneficial effects of green tea on oral health; ${ }^{21-26}$ however, the results are inconsistent.

Given the beneficial effects of green tea on oral health, as well as remineralization and antibacterial features of xylitol, the aim of this study was, therefore, to compare the effect of the "green tea with xylitol" mouthwash and the "green tea" mouthwash on the level of salivary S. mutans and Lactobacillus in children aged 6-12 years.

\section{Materials and Methods}

\section{Study Participants}

This double-blind randomized controlled clinical trial was performed in Isfahan, Iran, from January 2016 to September 2016. A total of 64 children aged 6-12 years ( 35 girls and 29 boys) were selected from the pediatric clinic of Isfahan Dental School. The sample size of the study was calculated based on the suggested formula for parallel clinical trials. By considering a type 1 error of $5 \%$, a type 2 error of $20 \%$ (power $=80 \%$ ), and $d=0.75$, we calculated a sample size of 54 subjects for the study. Considering the high rate of dropouts in clinical trials, 64 children were enrolled in this study. Participants were randomly assigned to "green tea" $(n=32)$ or "green tea with xylitol" ( $n=32$ ) mouthwash groups, each for a period of 2 weeks in a parallel design. Children and their parents were informed about the study procedure. The study was approved by the Ethical Committee of Isfahan University of Medical Sciences, Isfahan, Iran (grant \#390013). A written informed consent was obtained from all parents before participation in the study.

The inclusion criteria for entering the study were as follow: proper oral hygiene (brushing twice a day); lack of any untreated active carious lesions; absence of gingival or periodontal disease; no use of systemic antibiotics or fluoride therapy within the last 4 weeks; no habit of chewing xylitol-containing gum on a regular basis or drinking tea, coffee, or cacao; absence of any systemic diseases; absence of orthodontic appliances; and lack of any active abscess or drainage, with at least two decayed-missed-filled primary teeth (dmft). Participants were asked not to take xylitol chewing gum, systemic antibiotics, topical fluoride, tea, coffee, and cocoa during the study period and to report any changes in their general health status and medication.

\section{Study Design}

Participants were randomly divided into two groups in terms of the initial S. mutans count. Following saliva sample collection, the S. mutans colonies were measured. All children were randomly assigned to two groups. Information about each participant's

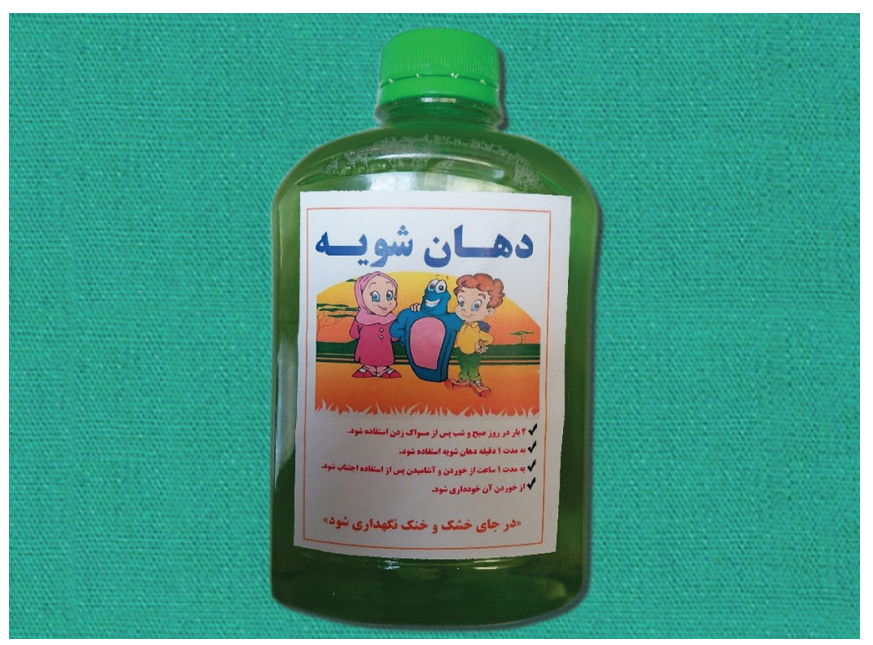

Fig. 1: A sample of mouthwashes used in the study

health, medication, dental care, and habits was recorded twice using a questionnaire.

After microbiological assessment, 0.5\% "green tea" and 20\% "green tea with xylitol" mouthwashes were given to the two groups, respectively. Mouthwashes were presented in $350 \mathrm{~mL}$ bottles. To make bottles more attractive for children, they were labeled with animation pictures and direction of use (Fig. 1). Participants in the "green tea" mouthwash group were asked to wash their mouth after brushing with $20 \mathrm{~mL}$ of "green tea" mouthwash for 60 seconds twice a day in the morning and at night. After each application, they were asked to refrain from eating or drinking for 1 hour. Participants in the "green tea with xylitol" mouthwash group were asked to rinse their teeth with $20 \%$ "green tea with xylitol" mouthwash for 2 minutes twice a day. Saliva samples were gathered 24 hours after the application of "green tea" and "green tea with xylitol" mouthwashes by participants for 2 weeks, and the level of S. mutans and Lactobacilli was determined. All participants were given the same toothbrush and fluoride (1100 ppm) toothpaste to brush their teeth twice a day during the study.

\section{Preparation of Mouthwashes}

To prepare $0.5 \%$ "green tea" mouthwash, $1.66 \mathrm{~L}$ green tea extract containing $6 \%$ phenolic compound was diluted with doubledistilled water to $20 \mathrm{~L}$. In order to produce $0.5 \%$ "green tea combined with $20 \%$ xylitol" mouthwash, $20 \%$ xylitol was added to the same mouthwash ( $200 \mathrm{~g} / \mathrm{L}$, equivalent to $20 \%)$. Finally, edible mint color $(20 \mathrm{~g} / \mathrm{L})$, mint flavor $(5.4 \mathrm{~mL} / \mathrm{L})$, and aspartame sweetener $(4 \mathrm{~g} / \mathrm{L})$ were added to the solution. ${ }^{27}$

\section{Saliva Sampling}

Since salivary microbial count is more stable than plaque, ${ }^{28,29}$ saliva samples were collected instead of plaques. Saliva sampling was performed before and after intervention. By simultaneous sampling of saliva (from 7.30 to 8.30, 1 hour after breakfast and prior to brushing), the probable variation in the saliva microbial count, which usually occurs during the day, ${ }^{30}$ was controlled. The saliva sample was taken by placing a sterile cotton at the mouth floor (sublingual) for 5 minutes and transferring it to $10 \mathrm{~mL}$ sterile tubes immediately. All saliva samplings were taken by a calibrated operator. The oral health of participants was assessed in accordance with the WHO criteria, ${ }^{31}$ and the number of dmft was recorded. 


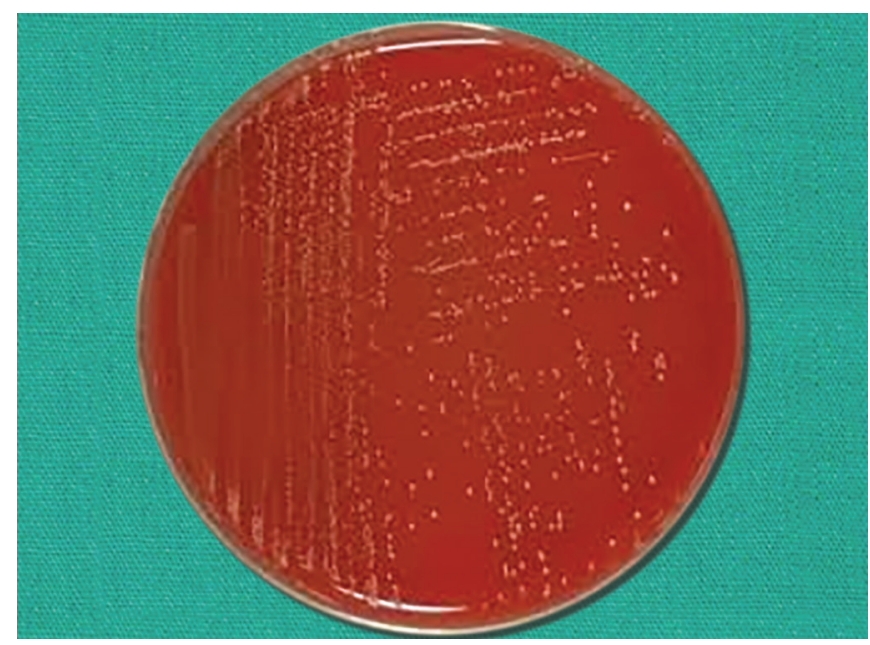

Fig. 2: Mitis salivarius culture media

\section{Microbial Analysis}

Microbial analysis was performed up to 45 minutes after the sample collection. To count S. mutans, $20 \mu \mathrm{L}$ of the saliva sample was spread on mitis salivarius agar (Difco) containing $0.2 \mathrm{unit} / \mathrm{mL}$ of bacitracin and sucrose $(15 \% \mathrm{w} / \mathrm{v}){ }^{32}$ Also, to measure the number of Lactobacilli colonies, $20 \mu \mathrm{L}$ of the saliva sample was spread on Rogosa agar (Unipath, Basingstoke, UK). The two groups of plates were incubated anaerobically $\left(85 \% \mathrm{~N}_{2}, 5 \% \mathrm{CO}_{2}\right.$, and $\left.10 \% \mathrm{H}_{2}\right)$ at $37^{\circ} \mathrm{C}$ for 3 days. Colony-forming units (CFUs) were identified based on morphology, size, and color and then counted by a stereomicroscope (Figs 2 and 3 ).

\section{Statistical Analysis}

The bacterial count in each group before and after intervention was compared using a paired $t$ test. An independent $t$ test was used to compare the two groups before and after the intervention. All analyses were performed using the SPSS version 22 software (SPSS Inc., Chicago, IL, USA). $p$ values less than 0.05 were considered statistically significant.

\section{Results}

The mean age of participants was $10.73 \pm 2.82$ years. At the beginning of the study, the average colony count of $S$. mutans in the total sample was $222 \pm 93.4$ (204.24 \pm 87.04 in the "green tea with xylitol" mouthwash group and $239.9 \pm 97.6$ in the "green tea" mouthwash group). According to the $t$ test analysis, there was no significant difference between the two groups in terms of CFUs of S. mutans prior to the intervention $(p=0.14)$.

After intervention, the mean number of colonies was $75.87 \pm 56$ in the "green tea with xylitol" mouthwash group and $146.4 \pm 69.6$ in the "green tea" mouthwash group. The results of the $t$ test analysis showed a significant difference between the two groups ( $p=0.001)$.

Overall, the mean reduction in the colony counts of $S$. mutans was $52 \pm 23 \%(65 \pm 18 \%$ in the "green tea with xylitol" mouthwash and $38 \pm 18 \%$ in the "green tea" mouthwash group), which was significantly higher in the "green tea with xylitol" mouthwash group compared with the "green tea" mouthwash group (Table 1).

In this study, 22 children (34.4\%) were aged less than 10 and 42 children (65\%) were aged greater than or equal to 10 years. The percentage of reduction in colony counts was $49.24 \pm 25.64 \%$ in children aged less than 10 years and $53.18 \pm 21 \%$ in children greater

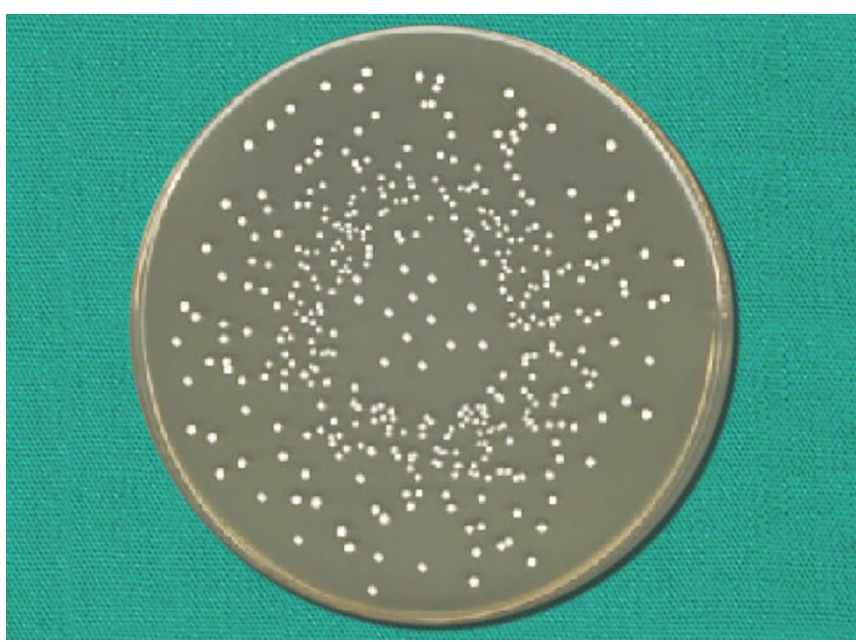

Fig. 3: Rogosa agar culture media

Table 1: Changes in S. mutans colony count (mean \pm standard deviation) in each group before and after intervention

\begin{tabular}{llcl}
\hline Intervention groups & $\begin{array}{l}\text { Green tea with } \\
\text { xylitol }\end{array}$ & Green tea & p value \\
\hline $\begin{array}{l}\text { Before intervention } \\
\text { (CFU/mL) }\end{array}$ & $204.87 \pm 2.04$ & $239.9 \pm 97.6$ & 0.14 \\
$\begin{array}{l}\text { After intervention } \\
\text { (CFU/mL) }\end{array}$ & $75.56 \pm 87$ & $146.69 \pm 4.6$ & 0.001 \\
\hline
\end{tabular}

CFU, colony-forming unit

Table 2: Changes in S. mutans colony count (mean \pm standard deviation) stratified by age

\begin{tabular}{|c|c|c|c|}
\hline Intervention groups & $\begin{array}{l}\text { Green tea with } \\
\text { xylitol }\end{array}$ & Green tea & $p$ value \\
\hline$<10$ years $(\mathrm{CFU} / \mathrm{mL}), \%$ & $20 \pm 68.7$ & $17.8 \pm 63.68$ & 0.49 \\
\hline$\geq 10$ years $(\mathrm{CFU} / \mathrm{mL}), \%$ & $15.8 \pm 31.9$ & $18.6 \pm 42.12$ & 0.14 \\
\hline
\end{tabular}

$\mathrm{CFU}$, colony-forming unit

Table 3: Changes in S. mutans colony count (mean \pm standard deviation) stratified by gender

\begin{tabular}{|c|c|c|c|}
\hline Intervention groups & $\begin{array}{l}\text { Green tea with } \\
\text { xylitol }\end{array}$ & Green tea & $p$ value \\
\hline Boys (CFU/mL), \% & $11.78 \pm 57.65$ & $16.3 \pm 72.1$ & 0.029 \\
\hline Girls (CFU/mL), \% & $16.27 \pm 35.46$ & $19.53 \pm 40.62$ & 0.45 \\
\hline
\end{tabular}

CFU, colony-forming unit

than or equal to 10 years. According to the $t$ test, there was no significant difference between the two age groups. The decreased number of colonies was not significant in any of the two age groups. In addition, no significant difference was observed between the two age groups in terms of colony count reduction percentage (Table 2).

The reduction in the number of colonies in boys and girls was $46.97 \pm 18.9$ and $55.87 \pm 25.42 \%$, respectively, and there was no significant difference between the two groups in this regard ( $p=$ 0.13 ). However, the percentage of variation in the number of the $S$. mutans colony between the two groups of "green tea with xylitol" and "green tea" mouthwashes was significantly different for boys ( $p=0.029$ ) (Table 3). 
Lactobacillus growth was observed in three subjects belonging to the "green tea with xylitol" mouthwash group and one belonging to the "green tea" mouthwash group, but this difference was not statistically significant.

\section{Discussion}

In this study, the effects of "green tea" and "green tea with xylitol" on the count of $S$. mutans and salivary Lactobacilli in children aged 6-12 years were evaluated. We observed that the "green tea with xylitol" mouthwash induced a significant reduction of S. mutans.

Green tea has always been known for its multifunctional clinical features, including its effect on decreasing the risk of cardiovascular diseases, stroke, obesity, and cancer. ${ }^{16-19}$ Studies have shown that bioactive compounds in green tea can affect the formation of caries. It can prevent tooth decay by preventing the proliferation of streptococcal agents, interfering with the enamel bonding process, or inhibiting the glycosyltransferase bacteria enzyme. ${ }^{20}$

Xylitol reduces the levels of $S$. mutans in plaques and saliva by disrupting the $S$. mutans energy production process, resulting in a futile cycle of energy and cell death. It also reduces the adhesion of microorganisms to the surface of teeth and diminishes their acid production potential. $^{33}$

In this study, the green tea mouthwash also resulted in a significant decrease in the number of $S$. mutans in saliva. Our results are consistent with findings of previous studies on the effect of green tea on decay bacteria. Xiao et al. showed that green tea polyphenols significantly inhibit the growth of S. mutans and Lactobacillus. ${ }^{20}$ By changing the bacterial phenotype, these compounds prevent its binding to the tooth surface. ${ }^{34}$ It has also been shown that the catechins in the green tea inhibit the glucosyltransferase enzyme, leading to a definite reduction of the plaque index..$^{20}$

The results of this study showed a significant difference between the effect of the "green tea with xylitol" mouthwash and the "green tea" mouthwash on S. mutans of saliva, which is higher in the "green tea with xylitol" group. Since the basis of mouthwashes used was similar in terms of solvent, flavor, and color, and was only different in terms of the effective ingredient (xylitol), the possibility of the effect of mouthwashes based on the amount of bacteria were eliminate.

Li et al. in an in vitro study showed that green tea polyphenols had no effect on the enamel remineralization of teeth and the anti-decay properties of this substance relate to its antibacterial action. ${ }^{23}$ However, further studies on the effect of polyphenols on enamel remineralization are required. On the other hand, another study demonstrated that the effect of $0.61 \%$ green tea solution on erosion and abrasion of dentin was not significantly different from fluoride. In fact, green tea polyphenols, especially catechins, cause the inhibition of the metalloproteinase matrix enzyme, which is responsible for the destruction of the dentin collagen matrix in carious lesions. ${ }^{35}$ Therefore, this substance can delay the dental caries process. Further studies in this area are needed to shed further light on this issue.

Like any other sweetener, xylitol increases mineralization by increasing the saliva flow when using gum or xylitol. Being unfermentable by the mouth bacteria is a unique feature of xylitol. Also, continuous use of xylitol leads to a reduction in S. mutans and plaque levels. ${ }^{33}$ Xylitol also promotes deeper layers of demineralized enamel by facilitating the movement of calcium and increasing its access, improving the mouthwash taste. ${ }^{15}$
Matsuyama et al. in another study on children aged 6-16 years indicated that daily consumption of catechin-rich green tea had no side effects on children's health. In addition, green tea led to weight loss and a decreased risk of cardiovascular disease in obese children. ${ }^{36}$ Other studies on the safety of catechins have not reported any specific side effects. ${ }^{37-39}$ It has been shown that the early deployment of $S$. mutans in children's mouths results in a higher incidence of caries at age 4. In fact, rapid transmission of S. mutans is associated with a higher chance of developing caries at older ages. ${ }^{40,41}$ Therefore, the use of a safe agent in childhood plays a major role in preventing dental caries in adulthood.

Our study has some limitations including the small sample size. It is recommended to conduct further studies with a larger sample size and longer duration of intervention to reach a definite conclusion.

\section{CONCLUSION}

The results of the present study showed that the "green tea with xylitol" mouthwash caused a significant reduction in the number of $S$. mutans in saliva, which is significantly higher than that of the "green tea" mouthwash.

\section{ACKNOWLEDgments}

The authors would like to thank all participants of the study for their great cooperation.

\section{Presentation at a Meeting}

This article has not been presented or published elsewhere.

\section{References}

1. Hardie JM, Whiley RA. Plaque microbiology of crown caries. In: Newman HN, Wilson M. ed. Dental plaque revisited Cardiff, Bioline; 1999. pp. 283-429.

2. Beighton D, Brailsford SR. Plaque microbiology of root caries. In: Newman HN, Wilson M. ed. Dental plaque revisited Cardiff, Bioline; 1999. pp. 295-312.

3. Ansai T, Tahara A, et al. Influence of colonization with mutans streptococci on caries risk in Japanese preschool children: 24 month survival analysis. Pediatr Dent 2000;22(5):377-380.

4. Berkowitz RJ, Jordan HV, et al. The early establishment of Streptococcus mutans in the mouths of infants. Arch Oral Biol 1975;20(3):171-174. DOI: 10.1016/0003-9969(75)90005-9.

5. Lopez L, Berkowitz R, et al. Topical antimicrobial therapy in the prevention of early childhood caries: a follow-up report. Pediatr Dent 2002;24(3):204-206.

6. Hamilton-Miller JM. Anti-cariogenic properties of tea (Camellia sinensis). J Med Microbiol 2001;50(4):299-302. DOI: 10.1099/00221317-50-4-299.

7. Lobo $\mathrm{PL}$, de Carvalho $\mathrm{CB}$, et al. Sodium fluoride and chlorhexidine effect in the inhibition of mutans streptococci in children with dental caries: a randomized, double-blind clinical trial. Oral Microbiol Immunol 2008;23(6):486-491. DOI: 10.1111/j.1399-302X.2008.00458.x.

8. Amin MS, Harrison RL, et al. Effect of povidone-iodine on Streptococcus mutans in children with extensive dental caries. Pediatr Dent 2004;26(1):5-10.

9. De Paola PF, Jordan HV, et al. Temporary suppression of Streptococcus mutans in humans through topical application of vancomycin. J Dent Res 1974;53(1):108-114. DOI: 10.1177/00220345740530010201.

10. Söderling E, Hirvonen A, et al. The Effect of Xylitol on the Composition of the Oral Flora: A Pilot Study. Eur J Dent 2011;5(1):24-31. 
11. Milgrom $P$, Ly KA, et al. Mutans streptococci dose response to xylitol chewing gum. J Dent Res 2006;85(2):177-181. DOI: 10.1177/154405910608500212.

12. Tanzer JM, Thompson A, et al. Streptococcus mutans: fructose transport, xylitol resistance, and virulence. J Dent Res 2006;85(4): 369-373. DOI: 10.1177/154405910608500417.

13. Mickenautsch S, Leal SC, et al. Sugar-free chewing gum and dental caries: a systematic review. J Appl Oral Sci 2007;15(2):83-88. DOI: 10.1590/s1678-77572007000200002.

14. Ritter AV, Preisser JS, et al. Risk indicators for the presence and extent of root caries among caries-active adults enrolled in the Xylitol for Adult Caries Trial (X-ACT). Clin Oral Investig 2012;16(6):1647-1657. DOI: 10.1007/s00784-011-0656-2.

15. Thabuis C, Cheng CY, et al. Effects of maltitol and xylitol chewinggums on parameters involved in dental caries development. Eur $J$ Paediatr Dent 2013;14(4):303-308.

16. Hertog MG, Feskens EJ, et al. Dietary antioxidant flavonoids and risk of coronary heart disease: the Zutphen Elderly Study. Lancet 1993;342(8878):1007-1011. DOI: 10.1016/0140-6736(93) 92876-u.

17. Keli SO, Hertog MG, et al. Dietary flavonoids, antioxidant vitamins, and incidence of stroke: the Zutphen study. Arch Intern Med 1996;156(6):637-642.

18. Bell SJ, Goodrick GK. A functional food product for the management of weight. Crit Rev Food Sci Nutr 2002;42(2):163-178. DOI: 10.1080/10408690290825501.

19. McKay DL, Blumberg JB. The role of tea in human health: an update. J Am Coll Nutr 2002;21(1):1-13. DOI: 10.1080/07315724.2002. 10719187.

20. Xiao Y, Liu T, et al. The effects of tea polyphenols on the adherence of cariogenic bacterium to the salivary acquired pellicle in vitro. Hua Xi Kou Qiang Yi Xue Za Zhi 2000;18(5):336-339.

21. Tehrani MH, Asghari G, et al. Comparing Streptococcus mutans and Lactobacillus colony count changes following green tea mouth rinse or sodium fluoride mouth rinse use in children (Randomized doubleblind controlled clinical trial). Dent Res J (Isfahan) 2011;8(Suppl 1): S58-S63.

22. Thomas A, Thakur SR, et al. Anti-microbial efficacy of green tea and chlorhexidine mouth rinses against Streptococcus mutans, Lactobacilli spp. and Candida albicans in children with severe early childhood caries: a randomized clinical study. J Indian Soc Pedod Prev Dent 2016;34(1):65-70. DOI: 10.4103/0970-4388.175518.

23. Li JY, Zhan L, et al. Effect of tea polyphenol on the demineralization and remineralization of enamel in vitro. Journal of Sichuan University Med Sci ed 2004;35(3):364-366.

24. Cardoso JG, lorio NL, et al. Influence of a Brazilian wild green propolis on the enamel mineral loss and Streptococcus mutans' count in dental biofilm. Arch Oral Biol 2016;65:77-81. DOI: 10.1016/ j.archoralbio.2016.02.001.
25. Cardoso CA, de Castilho AR, et al. Effect of xylitol varnishes on remineralization of artificial enamel caries lesions in vitro. J Dent 2014;42(11):1495-1501. DOI: 10.1016/j.jdent.2014.08.009.

26. Cardoso CA, Cassiano LP, et al. Effect of xylitol varnishes on remineralization of artificial enamel caries lesions in situ. J Dent 2016;50:74-78.

27. HandBook of pharmaceutipical formulations: Liquid products/ Sarfaraz K. Niazi. Iran; 2004.

28. Kohler B, Pettersson BM, et al. Streptococcus mutans in plaque and saliva and the development of caries. Scand J Dent Res 1981;89(1): 19-25. DOI: 10.1016/j.jdent.2016.03.011.

29. Mundorff $S A$, Eisenberg $A D$, et al. Correlations between numbers of microflora in plaque and saliva. Caries Res 1990;24(5):312-317. DOI: 10.1159/000261289.

30. Bentley C, Crawford JJ, et al. Analytical and physiological variability of salivary microbial counts. J Dent Res 1988;67(11):1409-1413. DOI: 10.1177/00220345880670111001.

31. World Health Organization: Oral Health Surveys: Basic Methods, ed 4. Genova; 1997.

32. Gold OG, Jordan $\mathrm{H}$, et al. A selective medium for Streptococcus mutans. Arch Oral Biol 1973;18(11):1357-1364. DOI: 10.1016/00039969(73)90109-x.

33. Nayak PA, Nayak UA, et al. The effect of xylitol on dental caries and oral flora. Clin Cosmet Investig Dent 2014;6:89-94. DOI: 10.2147/ CCIDE.S55761.

34. Otake S, Makimura M, et al. Anticaries effects of polyphenolic compounds from Japanese green tea. Caries Res 1991;25(6):438-443. DOI: 10.1159/000261407.

35. Magalhães AC, Wiegand A, et al. Chlorhexidine and green tea extract reduce dentin erosion and abrasion in situ. J Dent 2009;37(12): 994-998.

36. Matsuyama T, Tanaka Y, et al. Catechin safely improved higher levels of fatness, blood pressure, and cholesterol in children. Obesity (Silver Spring) 2008;16(6):1338-1348.

37. Chow HH, Cai Y, et al. Pharmacokinetics and Safety of Green Tea Polyphenols after Multiple-Dose Administration of Epigallocatechin Gallate and Polyphenon E in Healthy Individuals. Clin Cancer Res 2003;9(9):3312-3319.

38. Isbrucker RA, Edwards JA, et al. Safety studies on epigallocatechin gallate (EGCG) preparations. Part 2: dermal, acute and short-term toxicity studies. Food Chem Toxicol 2006;44(5):636-650.

39. Lauten JD, Boyd L, et al. A clinical study:Melaleuca, Manuka, Calendula and green tea mouth rinse. Phytother Res 2005;19(11):951-957.

40. Köhler B, Andréen I. The earlier the colonization by mutans streptococci, the higher the caries prevalence at 4 years of age. Oral Microbiol Immunol 1988;3(1):14-17.

41. Caufield PW, Cutter GR, et al. Initial acquisition of mutans streptococci by infants: evidence for a discrete window of infectivity. J Dent Res 1993;72(1):37-45. 